\title{
Computation of the limiting distribution in queueing systems with repeated attempts and disasters
}

Revue française d'automatique, d'informatique et de recherche opérationnelle. Recherche opérationnelle, tome 33, nº 3 (1999), p. 371-382.

<http://www.numdam.org/item?id=RO_1999_33_3_371_0>

(C) AFCET, 1999, tous droits réservés.

L'accès aux archives de la revue « Revue française d'automatique, d'informatique et de recherche opérationnelle. Recherche opérationnelle » implique l'accord avec les conditions générales d'utilisation (http://www.numdam.org/ legal.php). Toute utilisation commerciale ou impression systématique est constitutive d'une infraction pénale. Toute copie ou impression de ce fichier doit contenir la présente mention de copyright.

\section{Numdam}

Article numérisé dans le cadre du programme

Numérisation de documents anciens mathématiques

http://www.numdam.org/ 


\title{
COMPUTATION OF THE LIMITING DISTRIBUTION IN QUEUEING SYSTEMS WITH REPEATED ATTEMPTS AND DISASTERS (*)
}

\author{
by J.R. Artalejo $\left({ }^{1}\right)$ and A. Gómez-Corral $\left({ }^{1}\right)$ \\ Communicated by Erol Gelenbe
}

\begin{abstract}
Single server queues with repeated attempts are useful in the modeling of computer and telecommunication systems. In addition, we consider in this paper the possibility of disasters. When a disaster occurs, all the customers present in the system are destroyed immediately. Using a regenerative approach, we derive a numerically stable recursion scheme for the state probabilities. This model can be employed to analyze the behaviour of a buffer in computers with virus infections.
\end{abstract}

Keywords: Disasters, G-networks, queueing theory, repeated attempts.

Résumé. - Les files d'attente avec tentatives répétées sont utiles dans la modélisation des ordinateurs et des systèmes de télécommunication. En outre, nous considérons dans cet article la possibilité de désastres. Quand un désastre survient, tous les clients présents dans le système sont immédiatement détruits. En utilisant une méthode régénérative, nous établissons un schéma de récursion stable pour les probabilités d'état. Ce modèle peut être utilisé pour analyser le comportement d'un "buffer » dans des ordinateurs infectés par des virus.

Mots clés : Désastres, G-réseaux, files d'attente, tentatives répétées.

\section{INTRODUCTION}

Queueing systems with repeated attempts are characterized by the fact that a customer finding all the servers busy upon arrival must leave the service area, but some time later will.come back to re-initiate the demand. Between trials, a customer is said to be in "orbit". These models arise in practical situations as making phone calls or computers competing to gain service from a central processor unit. We mention [22] where retrial queues are used as mathematical models of several computer systems: packet switching

(*) Received July 1997.

( $\left.{ }^{1}\right)$ Faculty of Mathematics, Department of Statistics and O.R., University Complutense of Madrid, Madrid 28040, Spain.

Recherche opérationnelle/Operations Research, 0399-0559/99/03/\$7.00

(C) EDP Sciences, 1999 
networks, shared bus local area networks operating under the Carrier-Sense Multiple Access protocol and collision avoidance star local area networks. Interested readers can find the main results and methods for retrial queues in the monograph by Falin and Templeton [6].

It should be pointed out that the evolution of a single server retrial queue exhibits an alternating sequence of idle and busy periods of the server. At any service completion epoch, the server becomes free. Then, a competition between the primary input flow and the repeated attempts corresponding to customers at the retrial group determines the next customer who accesses to the server. This is just the main difference with classical waiting lines without retrials. The second input stream associated to the repeated attempts is usually a non-homogeneous flow (see Sect. 2). The analytical difficulties arising in the study of retrial queues are connected with this lack of homogeneity.

A number of papers $[3-5,15]$ have recently appeared in the queueing literature in which a catastrophe removes all the work present in the system. These disasters can be view as a general breakdown of the system so these models can be used to analyze computer networks with virus infections, and breakdowns due to a reset order. Besides, the catastrophes can also be connected with other queueing phenomena such as G-networks and clearing systems [7-14, 19, 20].

G-networks were introduced in their simplest version by Gelenbe [9]. In this versatile class of queueing networks the customers are either "positive" or "negative". In contrast with the ordinary customers, the arrival of a negative customer implies that one positive customer must be removed, if any presents, from the system. Since their introduction, there has been a growing interest in several directions, including extensions to the case of multiple classes of customers [7, 13], networks with triggered customer motion [11], networks with batch and random amount of work removal $[4,12]$ and analysis of single node queues with negative customers [14].

On the other hand, the early work on stochastic clearing systems $[19,20]$ describes the behaviour of these models in terms of a stochastic input flow and an output mechanism which instantaneously deletes all backlog in the system. The literature shows applications to inventory systems, queueing theory and public service systems. Most efforts were done to investigate the optimal design of a clearing model when the clearing level is subject to control and a cost structure is assumed.

In this context, queueing networks with disasters provide an extreme case in which all the customers at the node are deleted automatically when a disaster occurs. We note that the concept of "disaster" is closely related to the 
models with batch removal. Following [12], it suffices to take the distribution of the batch size of node $i, P\left\{B_{i}=s\right\}=\Pi_{i s}, s \geq 1$, appropriately in order to remove all customers present at that node; i.e., we consider $P\left\{B_{i}=s\right\}$ as a non honest distribution and define $\Pi_{i s}=0$ for all finite $s$.

In this paper we study a single node queue with state dependent input and one server simultaneously allowing for repeated attempts and a disaster mechanism (see Sect. 2 for the model description). The particular case in which the input stream is an homogeneous Poisson process was introduced in [3] where the authors investigated the following features:

a) the joint distribution of the server state and the number of customers in orbit in steady state,

b) the joint distribution of a busy period and the number of service completions and deleted customers,

c) the waiting time for the case in which only the customer at the head of the retrial group is allowed for access to the server.

The analysis of point $a$ ) in [3] was given in terms of generating functions where complex integral formulae are involved. So our goal in this paper is to complete the investigation in a double sense:

i) by providing a stable scheme for computing the limiting distribution in a class of retrial queues which includes the system studied in [3],

ii) by reducing the numerical efforts for analyzing the main performance characteristics.

To that end, we will generalize a classical approach based on the theory of regenerative processes. Schellhaas [18] and Tijms [21] showed the usefulness of this approach in a general class of queueing models. The application of this methodology also leads to computationally tractable algorithms for computing the state probabilities in single server retrial queues $[1,16]$. Nevertheless, all this work has been done for single server queues where a matricial structure of type $M / G / 1$ is preserved. After a disaster, all the customers in the system are removed immediately; i.e., a transition to state $(0,0)$ occurs. From this point of view, we extend in this paper the applicability of the regenerative methodology to a more general context where the standard matricial structure is not preserved.

The rest of the paper is organized as follows. In Section 2 we describe the mathematical model. In Section 3 we derive the recursive algorithm for computing the limiting distribution of the system state. Finally, in Section 4 we give some numerical results. 


\section{THE MATHEMATICAL MODEL}

Let us consider a single server queueing system at which customers arrive according to a state dependent Markovian input process with rate $\lambda_{i j}$ when the system is in state $(i, j)$. The first component $i=0$ or 1 according as the server is free or busy and $j$ denotes the number of customers in the orbit. We will consider two different retrial policies for describing how the customers in orbit can access to the server. Most queueing systems with repeated attempts assume that each customer in orbit seeks service independently of each other after a random time exponentially distributed with rate $\mu$. Thus, the probability of a repeated attempt during the interval $(t, t+\Delta t)$, given that $j$ customers were in orbit at time $t$, is $j \mu \Delta t+o(\Delta t)$. However, there is a second retrial policy where the retrial rate does not depend on the number of customers in orbit (if any), so the probability of a repeated attempt during $(t, t+\Delta t)$, given that the orbit is not idle at time $t$, is $\mu \Delta t+o(\Delta t)$. The $M / G / 1$ retrial queue with the latter policy was studied extensively in [17]. In what follows we will incorporate both retrial policies by assuming the linear retrial policy introduced in [2]; i.e., the probability of a repeated attempt during the interval $(t, t+\Delta t)$, given that $j$ customers were in orbit at time $t$, is $\left(\alpha\left(1-\delta_{0 j}\right)+j \mu\right) \Delta t+o(\Delta t)$, where $\delta_{a b}$ denotes Kronecker's delta. The service times of customers are independent and distributed as a random variable $S$ having probability distribution function $B(t)(B(0)=0)$ and Laplace-Stieltjes transform $\beta(\theta)=E[\exp \{-\theta S\}]$. Besides, the disasters also arrive at the system according to a Poisson process with rate $\delta$. If a disaster occurs then all the customers in the system are immediately destroyed. The input streams of primary arrivals and disasters, service times and intervals between successive repeated attempts are assumed to be mutually independent.

The state dependent Markovian input process covers a number of important particular cases in practice. Taking $\lambda_{i j}=\lambda$, for $i \in\{0,1\}$ and $j \geq 0$, we have the $M / G / 1$ queue with linear retrial policy and disasters introduced in [3]. If we assume a model with finite orbit capacity $K$ then the arrival intensities are taken as $\lambda_{i j}=\lambda$, for $i \in\{0,1\}, 0 \leq j \leq K-1, \lambda_{0 K}=\lambda$ and $\lambda_{i j}=0$ otherwise. Taking $\lambda_{i j}=(K-i-j) \lambda$, for $(i, j)$ such that $0 \leq i+j \leq K$ we have the quasi-input process used to model the repairman problem with $K$ machines.

Observe that the existence of a flow of disasters with positive rate $\delta$ guarantees that the limiting distribution always exists. It should be pointed out that the analysis of Section 3 will be given for finite and infinite orbit capacity systems simultaneously. 


\section{THE REGENERATIVE ANALYSIS AND THE RECURSIVE APPROACH}

The state of the system can be described by means of the process $(C(t), N(t), \xi(t))$ where $C(t)$ is 0 or 1 according as the server is free or busy at time $t, N(t)$ represents the number of customers in orbit and, if $C(t)=1$, then $\xi(t)$ represents the elapsed time of the customer being served. We next neglect $\xi(t)$ and consider only the bidimensional process $(C(t), N(t))$ with state space $E=\{0,1\} \times\{0, \ldots, K\}$.

Let $P_{i j}=\lim _{t \rightarrow \infty} P\{C(t)=i, N(t)=j\}$ be the limiting distribution of the system state. Let a cycle be the elapsed time between two successive visits of the process $(C(t), N(t))$ to the state $(0,0)$. We also define some other random variables:

$T=$ the length of a cycle,

$T_{i j}=$ the amount of time in a cycle during which $(C(t), N(t))=(i, j)$, $(i, j) \in E$,

$N_{0 j}=$ the number of service completions in a cycle at which $j$ customers are left behind in orbit, $j \geq 1$,

$N=$ the total number of service completions and disasters in a cycle.

We can also define $N_{00}$ as the number of events in a cycle at which 0 customers are left behind in orbit. Obviously, $N_{00}$ equals 1 but this event may be either an ordinary service completion or a disaster.

From the theory of regenerative processes, we have

$$
P_{i j}=\frac{E\left[T_{i j}\right]}{E[T]}, \quad \text { for all }(i, j) \in E
$$

The keys for developing an algorithmic method for computing the limiting distribution are the equation (1) and some up and down crossing arguments. The number of transitions from state $(0, j)$ is equal to the number of transitions into $(0, j)$ in a regeneration cycle $(0, T]$. Then, by equating the corresponding expectations we obtain

$$
\left(\lambda_{0 j}+\alpha\left(1-\delta_{0 j}\right)+j \mu+\delta\left(1-\delta_{0 j}\right)\right) E\left[T_{0 j}\right]=E\left[N_{0 j}\right], \quad 0 \leq j \leq K .
$$

We further observe that the number of transitions in $(0, T]$ at which the orbit size increases from $j-1$ to $j$ equals the number of transitions at which the 
orbit size decreases from $j$ to $j-1$. Taking expectations the above argument leads to the equation

$$
\lambda_{1, j-1} E\left[T_{1, j-1}\right]=(\alpha+j \mu) E\left[T_{0 j}\right]+\delta \sum_{n=j}^{K}\left(E\left[T_{0 n}\right]+E\left[T_{1 n}\right]\right), \quad 1 \leq j \leq K .
$$

Note that (3) is equivalent to

$$
\begin{gathered}
\lambda_{1, j-1} E\left[T_{1, j-1}\right]+\delta \sum_{n=0}^{j-1}\left(E\left[T_{0 n}\right]+E\left[T_{1 n}\right]\right)=(\alpha+j \mu) E\left[T_{0 j}\right]+\delta E[T], \\
1 \leq j \leq K .
\end{gathered}
$$

Dividing (4) by $E[T]$ we have from (1) that

$$
\lambda_{1, j-1} P_{1, j-1}+\delta \sum_{n=0}^{j-1}\left(P_{0 n}+P_{1 n}\right)=(\alpha+j \mu) P_{0 j}+\delta, \quad 1 \leq j \leq K .
$$

Hence the computation of the limiting distribution is reduced to find the sequence $\left\{P_{1 j}\right\}_{j=0}^{K}$, since $P_{00}$ can be obtained from

$$
P_{00}=1-P_{10}-\sum_{n=1}^{K}\left(P_{0 n}+P_{1 n}\right)
$$

To derive a recurrence relation among the probabilities $P_{1 j}$, we define the following quantity. For $0 \leq j \leq K$ and $0 \leq k \leq \min (j+1, K)$ let

$A_{k j}=$ the expected amount of time that during a service time $j$ customers are in the orbit given that the previous service time (or disaster) left $k$ customers in orbit.

Observe that a straightforward application of Wald's theorem yields

$$
E\left[T_{1 j}\right]=\sum_{k=0}^{\tilde{j}+1} A_{k j} E\left[N_{0 k}\right], \quad 0 \leq j \leq K,
$$

where $\tilde{j}=\min (j, K-1)$.

From (2) we rewrite (7) as follows

$$
E\left[T_{1 j}\right]=A_{0 j}+\sum_{k=1}^{\tilde{j}+1} A_{k j}\left(\lambda_{0 k}+\alpha+k \mu+\delta\right) E\left[T_{0 k}\right], \quad 0 \leq j \leq K .
$$


Next using (4) and (8), we obtain

$$
\begin{aligned}
& E\left[T_{1 j}\right]=\left(A_{0 j}+\left(1-\delta_{0 j}\right) \sum_{k=1}^{j} \lambda_{1, k-1}\left(1+\frac{\lambda_{0 k}+\delta}{\alpha+k \mu}\right) A_{k j} E\left[T_{1, k-1}\right]\right. \\
&+\delta\left(\left(1-\delta_{j K}\right)\left(1+\frac{\lambda_{0, j+1}+\delta}{\alpha+(j+1) \mu}\right) A_{j+1, j} E\left[T_{0 j}\right]\right. \\
&+\left(1-\delta_{0 j}\right) \sum_{n=0}^{j-1}\left(E\left[T_{0 n}\right]+E\left[T_{1 n}\right]\right) \\
&\left.\left.\times \sum_{k=n+1}^{\tilde{j}+1}\left(1+\frac{\lambda_{0 k}+\delta}{\alpha+k \mu}\right) A_{k j}-E[T] \sum_{k=1}^{\tilde{j}+1}\left(1+\frac{\lambda_{0 k}+\delta}{\alpha+k \mu}\right) A_{k j}\right)\right) \\
& \times\left(1-\left(1-\delta_{j K}\right)\left(\lambda_{1 j}+\delta\right)\left(1+\frac{\lambda_{0, j+1}+\delta}{\alpha+(j+1) \mu}\right) A_{j+1, j}\right)^{-1}, \\
& 0 \leq j \leq K .
\end{aligned}
$$

Dividing both sides of (9) by $E[T]$ and using the fact that $E[T]=$ $\left(\lambda_{00} P_{00}\right)^{-1}$, we find that

$$
\begin{aligned}
& P_{1 j}=\left(\lambda_{00} A_{0 j} P_{00}+\left(1-\delta_{0 j}\right) \sum_{k=1}^{j} \lambda_{1, k-1}\left(1+\frac{\lambda_{0 k}+\delta}{\alpha+k \mu}\right) A_{k j} P_{1, k-1}\right. \\
&+\delta\left(\left(1-\delta_{j K}\right)\left(1+\frac{\lambda_{0, j+1}+\delta}{\alpha+(j+1) \mu}\right) A_{j+1, j} P_{0 j}\right. \\
&+\left(1-\delta_{0 j}\right) \sum_{n=0}^{j-1}\left(P_{0 n}+P_{1 n}\right) \\
&\left.\left.\times \sum_{k=n+1}^{\tilde{j}+1}\left(1+\frac{\lambda_{0 k}+\delta}{\alpha+k \mu}\right) A_{k j}-\sum_{k=1}^{j+1}\left(1+\frac{\lambda_{0 k}+\delta}{\alpha+k \mu}\right) A_{k j}\right)\right) \\
& \times\left(1-\left(1-\delta_{j K}\right)\left(\lambda_{1 j}+\delta\right)\left(1+\frac{\lambda_{0, j+1}+\delta}{\alpha+(j+1) \mu}\right) A_{j+1, j}\right)^{-1} \\
& 0 \leq j \leq K .
\end{aligned}
$$

An alternating application of formulas (5) and (10) provides a stable recursive scheme for computing the limiting probabilities in terms of $P_{00}$ which is given by expression (6). 
It remains to specify the calculation of the quantities $A_{k j}$. To that end we now define a second auxiliary quantity $B_{k j}$. For $0 \leq k \leq j \leq K$ let

$B_{k j}=$ the expected amount of time that during a service time $j$ customers are in orbit given that immediately after the beginning of the service $k$ customers were in orbit.

We next find that these quantities are given by

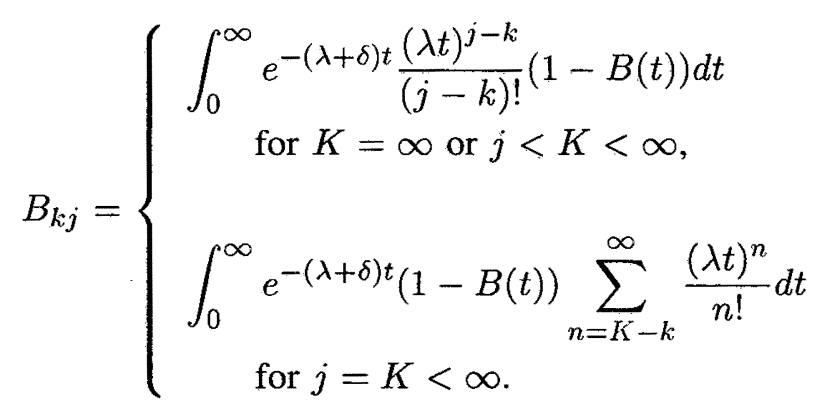

We now verify the validity of $B_{k j}$ in case that $K=\infty$ or $j<K<\infty$. If we consider an infinitesimal interval $(t, t+\Delta t)$, then the interval contributes to $B_{k j}$ if the service time has not been completed before time $t$ (with probability $1-B(t)$ ), a disaster did not occur (with probability $e^{-\delta t}$ ) and $j-k$ customers arrived in $(0, t)$ (with probability $e^{-\lambda t}(\lambda t)^{j-k} /(j-k) !$ ). The case $j=K<\infty$ follows along the same lines. Now there must be at least $K-k$ arrivals in the interval $(0, t)$.

Then, by connecting $A_{k j}$ and $B_{k j}$, we obtain

$$
\begin{gathered}
A_{k j}=\left(\alpha\left(1-\delta_{0 k}\right)+k \mu\right) \gamma_{k} B_{k-1, j}+\lambda_{0 k} \gamma_{k} B_{k j}, \quad 0 \leq k \leq j \leq K, \\
A_{j+1, j}=(\alpha+(j+1) \mu) \gamma_{j+1} B_{j j}, \quad 0 \leq j \leq K-1,
\end{gathered}
$$

where

$$
\gamma_{k}=\left(\lambda_{0 k}+\alpha\left(1-\delta_{0 k}\right)+k \mu+\delta\left(1-\delta_{0 k}\right)\right)^{-1}, \quad k \geq 0 .
$$

In Section 4 we will show that the computational work introduced by the integral in (11) may be reduced to a finite sum for most service time distributions of practical interest.

\section{NUMERICAL EXAMPLES}

In this section we consider several examples in which computationally tractable expressions for the quantities $B_{k j}$ can be given. 
Example 1. Exponential service time distribution. Now we have

$$
B(t)=1-e^{-\nu t}, \quad t>0 .
$$

Hence

$$
B_{k j}= \begin{cases}\frac{1}{\lambda+\nu+\delta}\left(\frac{\lambda}{\lambda+\nu+\delta}\right)^{j-k} & \text { for } K=\infty \text { or } j<K<\infty, \\ \frac{1}{\nu+\delta}\left(\frac{\lambda}{\lambda+\nu+\delta}\right)^{K-k} & \text { for } j=K<\infty .\end{cases}
$$

Example 2. Deterministic service time distribution. Assume that the service time of a customer is a constant $D>0$. Then it is easy to show that

$$
B_{k j}=\left\{\begin{array}{l}
\frac{1}{\lambda+\delta}\left(\frac{\lambda}{\lambda+\delta}\right)^{j-k}\left(1-e^{-(\lambda+\delta) D} \sum_{n=0}^{j-k} \frac{((\lambda+\delta) D)^{n}}{n !}\right) \\
\quad \text { or } K=\infty \text { or } j<K<\infty, \\
\delta^{-1}\left(\left(\frac{\lambda}{\lambda+\delta}\right)^{K-k}-e^{-\delta D}-\left(1-\delta_{k K}\right) e^{-(\lambda+\delta) D}\right. \\
\left.\times\left(\left(\frac{\lambda}{\lambda+\delta}\right)^{K-k} \sum_{n=0}^{K-k-1} \frac{((\lambda+\delta) D)^{n}}{n !}-\sum_{n=0}^{K-k-1} \frac{(\lambda D)^{n}}{n !}\right)\right) \\
\quad \text { for } j=K<\infty .
\end{array}\right.
$$

Example 3. Coxian-2 service time distribution. Suppose the density function defined as follows

$$
b(t)=p_{1} \nu_{1} e^{-\nu_{1} t}+p_{2} \nu_{2} e^{-\nu_{2} t}, \quad t>0,
$$

where $\nu_{1}>\nu_{2}>0, p_{2}=1-p_{1}$ and $p_{1}=1-b \nu_{1} /\left(\nu_{1}-\nu_{2}\right)$. The parameter $b$ belongs to the interval $[0,1]$. A Coxian- 2 distributed random variable $X$ has a coefficient of variation greater than $1 / 2$. In this case $B_{k j}$ can be reduced to the following expressions

$$
B_{k j}= \begin{cases}\sum_{n=1}^{2} \frac{p_{n}}{\lambda+\nu_{n}+\delta}\left(\frac{\lambda}{\lambda+\nu_{n}+\delta}\right)^{j-k} & \text { for } K=\infty \text { or } j<K<\infty, \\ \sum_{n=1}^{2} \frac{p_{n}}{\nu_{n}+\delta}\left(\frac{\lambda}{\lambda+\nu_{n}+\delta}\right)^{K-k} & \text { for } j=K<\infty\end{cases}
$$


TABLE 1

Limiting distribution of some retrial queues with disasters.

\begin{tabular}{|c|c|c|c|c|c|c|}
\hline \multirow[b]{2}{*}{$j$} & \multicolumn{2}{|c|}{$\begin{array}{l}\text { Exponential distribution } \\
(\lambda, \alpha, \mu, \nu, \delta, K)= \\
(4.7,1.4,5.5,5.3,0.001,19)\end{array}$} & \multicolumn{2}{|c|}{$\begin{array}{l}\text { Deterministic distribution } \\
(\lambda, \alpha, \mu, D, \delta, K)= \\
(0.75,4.3,0,0.3,1.1,12)\end{array}$} & \multicolumn{2}{|c|}{$\begin{array}{l}H_{2} \text { distribution } \\
\left(\lambda, \alpha, \mu, p_{1}, \nu_{1}, \nu_{2}, \delta, K\right)= \\
(5.3,0,2.9,0.25,2,4,0.3,17)\end{array}$} \\
\hline & $P_{0 j}$ & $P_{1 j}$ & $P_{0 j}$ & $P_{1 j}$ & $P_{0 j}$ & $P_{1 j}$ \\
\hline 0 & 0.028364 & 0.024970 & 0.803077 & 0.154681 & 0.090909 & 0.061364 \\
\hline 1 & 0.016871 & 0.036929 & 0.016173 & 0.020888 & 0.024453 & 0.062090 \\
\hline 2 & 0.013925 & 0.044931 & 0.002318 & 0.002306 & 0.017366 & 0.059921 \\
\hline 3 & 0.011751 & 0.050111 & 0.000260 & 0.000237 & 0.012921 & 0.056481 \\
\hline 4 & 0.010032 & 0.053190 & 0.000026 & 0.000024 & 0.009914 & 0.052525 \\
\hline 5 & 0.008625 & 0.054686 & 0.000002 & 0.000002 & 0.007777 & 0.048434 \\
\hline 6 & 0.007452 & 0.054984 & $0.27 \times 10^{-6}$ & $0.25 \times 10^{-6}$ & 0.006203 & 0.044410 \\
\hline 7 & 0.006462 & 0.054381 & $0.28 \times 10^{-7}$ & $0.25 \times 10^{-7}$ & 0.005015 & 0.040569 \\
\hline 8 & 0.005618 & 0.053109 & $0.29 \times 10^{-8}$ & $0.26 \times 10^{-8}$ & 0.004100 & 0.036978 \\
\hline 9 & 0.004894 & 0.051351 & $0.30 \times 10^{-9}$ & $0.27 \times 10^{-9}$ & 0.003387 & 0.033694 \\
\hline 10 & 0.004272 & 0.049250 & $0.30 \times 10^{-10}$ & $0.27 \times 10^{-10}$ & 0.002832 & 0.030783 \\
\hline 11 & 0.003733 & 0.046920 & $0.31 \times 10^{-11}$ & $0.30 \times 10^{-11}$ & 0.002407 & 0.028365 \\
\hline 12 & 0.003267 & 0.044449 & $0.40 \times 10^{-12}$ & $0.31 \times 10^{-12}$ & 0.002103 & 0.026672 \\
\hline 13 & 0.002862 & 0.041907 & & & 0.001932 & 0.026167 \\
\hline 14 & 0.002509 & 0.039349 & & & 0.001936 & 0.027773 \\
\hline 15 & 0.002202 & 0.036816 & & & 0.002208 & 0.033302 \\
\hline 16 & 0.001934 & 0.034339 & & & 0.002931 & 0.046297 \\
\hline 17 & 0.001699 & 0.031942 & & & 0.004455 & 0.081307 \\
\hline 18 & 0.001494 & 0.029640 & & & & \\
\hline 19 & 0.001315 & 0.027446 & & & & \\
\hline
\end{tabular}

In particular, the hyperexponential service time distribution $H_{2}$ requires that the weights $p_{1}$ and $p_{2}$ are non-negative.

Other many service time distributions (shifted exponential and mixture of Erlang distributions) also lead to closed form expressions for $B_{k j}$. Our numerical experience shows that the recursion scheme provided by formulas (5) and (10) is numerically stable. Note that the scheme only involves one difference in (10) and thus a loss of significant digits is not expected.

The model with infinity capacity of the orbit $\left(\lambda_{i j}=\lambda, i \in\{0,1\}, j \geq 0\right)$ must be reduced to a finite system by placing a ficticious limit on the number of customers in the orbit. This auxiliary limit, say $N$, can be chosen so that the probability $P_{00}$ has a desired accuracy. Nevertheless, a direct truncation over (5) and (10) leads to a finite system of equations which does not correspond to the limiting probabilities of any queueing model. Thus it is impossible to guarantee that $P_{00}$ belongs to the interval $(0,1)$. To solve this problem, we propose a simple approximation of the original infinite system by a finite orbit capacity system where $\lambda_{i j}=\lambda$, for $i \in\{0,1\}$, $0 \leq j \leq K-1, \lambda_{0 K}=\lambda$ and $\lambda_{i j}=0$ otherwise. The value of $K$ can be 
TABLE 2

Comparison between the solutions based on NRS and the regenerative approach.

\begin{tabular}{|c|c|c|}
\hline & Results based on NSR & $\begin{array}{c}\text { Results based } \\
\text { on regenerative approach } \\
P_{00}\end{array}$ \\
\hline 0.1 & $P_{00}$ & 0.173975 \\
0.2 & 0.174504 & 0.260094 \\
0.4 & 0.260508 & 0.368320 \\
0.6 & 0.368445 & 0.439448 \\
0.8 & 0.439471 & 0.491962 \\
1.0 & 0.491957 & 0.533175 \\
1.2 & 0.533164 & 0.566794 \\
1.4 & 0.566782 & 0.594972 \\
1.6 & 0.594958 & 0.619068 \\
\hline
\end{tabular}

determined with the help of the normalizing condition (6) at any desired accuracy level.

In Table 1 we give the limiting distribution for several retrial queues with disasters. The service time includes the exponential, deterministic and hyperexponential distributions. The retrial parameters have been chosen to cover the cases of classical, constant and linear repeated attempts.

In the case $K=\infty$ and $\lambda_{i j}=\lambda$ for all $(i, j) \in E$, the generating transforms and the main characteristics associated to the limiting distribution can be expressed in terms of complex integral solutions. We refer to the interested reader for seeing the expressions given in Theorem 1 and Corollary 1 in [3]. A numerical solution can be obtained by a Fortran program calling Numerical Recipes Software (NRS). Nevertheless, it is obvious that the numerical efforts for computing some performance measures can be simplyfied with the help of the regenerative approach developed in Section 3. From the point of view of accuracy, both methods provide similar results. This fact is illustrated in Table 2 for the probability $P_{00}$. The data correspond to the queue with hyperexponential service time distribution $\mathrm{H}_{2}$ and parameters $\left(\lambda, \alpha, \mu, p_{1}, \nu_{1}, \nu_{2}\right)=(1.6,2,0.7,0.3,2.7,1.5)$.

\section{ACKNOWLEDGEMENTS}

This work was supported by the DGICYT under PB95-0416 and the European Commission under INTAS 96-0828. The authors wish to thank the referee for his constructive comments. 


\section{REFERENCES}

1. J. R. Artalejo, New results in retrial queueing systems with breakdown of the servers, Statist. Neerlandica, 1994, 48, p. 23-36.

2. J. R. Artalejo and A. Gomez-Corral, Steady state solution of a single-server queue with linear request repeated, J. Appl. Probab., 1997, 34, p. 223-233.

3. J. R. Artalejo and A. Gomez-Corral, Analysis of a stochastic clearing system with repeated attempts, Communications in Statistics-Stochastic Models, 1998, 14, p. 623-645.

4. R. J. Boucherie and O. J. Boxma, The workload in the $M / G / 1$ queue with work removal, Probab. Engineering and Informational Sci., 1996, 10, p. 261-277.

5. X. CHAO, A queueing network model with catastrophes and product form solution, Operations Research Letters, 1995, 18, p. 75-79.

6. G. I. Falin and J. G. C. Templeton, Retrial Queues, Chapman and Hall, London, 1997.

7. J. M. Fourneau, E. Gelenbe and R. Suros, G-Networks with multiple classes of positive and negative customers, Theoret. Comput. Sci., 1996, 155, p. 141-156.

8. E. Gelenbe, P. Glynn and K. Sigman, Queues with negative arrivals, J. Appl. Probab., 1991, 28, p. 245-250.

9. E. Gelenbe, Queueing networks with negative and positive customers and product form solution, J. Appl. Probab., 1991, 28, p. 656-663.

10. E. Gelenbe and M. Schassberger, Stability of product form G-Networks, Probab. Engineering and Informational Sci., 1992, 6, p. 271-276.

11. E. Gelense, G-Networks with triggered customer movement, J. Appl. Probab., 1993, 30 , p. $742-748$.

12. E. Gelenbe, G-Networks with signals and batch removal, Probab. Engineering and Informational Sci., 1993, 7, p. 335-342.

13. E. Gelenbe and A. Labed, G-Networks with multiple classes of signals and positive customers, European J. Oper. Res., 1998, 108, p. 393-405

14. P. G. Harrison and E. Prtel, The $M / G / 1$ queue with negative customers, Adv. Appl. Probab., 1996, 28, p. 540-566.

15. G. JAIN and K. SIGMAN, A Pollaczek-Khintchine formula for $M / G / 1$ queues with disasters, J. Appl. Probab., 1996, 33, p. 1191-1200.

16. A. G.DE KoK, Algorithmic methods for single server systems with repeated attempts, Statist. Neerlandica, 1984, 38, p. 23-32.

17. M. Martin and J. R. Artalejo, Analysis of an $M / G / 1$ queue with two types of impatient units, Adv. Appl. Probab., 1995, 27, p. 840-861.

18. H. SchellhaAs, Computation of the state probabilities in a class of semiregenerative queueing Models, J. Janssen, Ed., Semi-Markov Models: Theory and Applications, Plenum Press, New York and London, 1986, p. 111-130.

19. R. Serfozo and S. Stidham, Semi-stationary clearing processes, Stochastic Process. Appl., 1978, 6, p. 165-178.

20. S. Stidham, Stochastic clearing systems, Stochastic Process. Appl., 1974, 2, p. 85-113.

21. H. C. TIJMs, Stochastic Models: An Algorithmic Approach, John Wiley and Sons, Chichester, 1994.

22. T. Yang and J. G. C. Templeton, A survey on retrial queues, Queueing Systems, 1987, 2, p. 201-233. 